\title{
Involvement of Prx3, a Drosophila ortholog of the thiol-dependent peroxidase PRDX3, in age-dependent oxidative stress resistance
}

Yasunari KaYashima and Kimiko YamaKaWa-KoBayashi

School of Food and Nutritional Sciences, Graduate School of Integrated Pharmaceutical and Nutritional Sciences, University of Shizuoka, Japan

(Received 22 August 2012; and accepted 24 August 2012)

\begin{abstract}
Peroxiredoxins (Prxs) are a family of multifunctional antioxidant thioredoxin-dependent peroxidases. We used Drosophila melanogaster to examine the function of Prx3, the Drosophila homolog of human PRDX3. The oxidative stress response in adult Drosophila is age-dependent. RNAiinduced Prx3 knockdown in adult flies did not change their phenotype in normal conditions, but they had a shorter survival than the $\operatorname{Prx} 3^{+}$controls in the presence of $\mathrm{H}_{2} \mathrm{O}_{2}$. The expression levels of the $\operatorname{Prx} 3$ were reduced by aging. These results suggest that Prx3 plays an important role in the oxidative stress response and is involved in the age-dependent competence of the oxidative stress response.
\end{abstract}

In multicellular organisms, the accumulation of reactive oxygen species (ROS) increases with age (10). This increase is considered to be attributed to its simple accumulation and to the impairment of the ROS scavenging capacity of the cell that is mainly due to dysfunction of the mitochondria, the major source of intracellular $\operatorname{ROS}(2,12,15)$. The incidence rates of cardiovascular diseases and neurodegenerative diseases also increase with age, indicating that ROS accumulation is a risk factor for age-related diseases $(5,14)$. Oxidative stress plays an important role in governing the life span of the fruit fly Drosophila melanogaster $(4,9)$. It is considered that responsiveness to oxidative stress declines with the number of days after eclosion. To determine whether the oxidative stress response alters with age in Drosophila, eclosed virgin male flies were isolated and kept in normal conditions for 2, 5 or 16 days, and then fed with food containing $0.5 \% \mathrm{H}_{2} \mathrm{O}_{2}$. We

Address correspondence to: Dr Yasunari Kayashima School of Food and Nutritional Sciences, Graduate School of Integrated Pharmaceutical and Nutritional Sciences, University of Shizuoka, 52-1 Yada, Suruga-ku, Shizuoka 422-8526, Japan Tel: +81-54-264-5226, Fax: +81-54-264-5220

E-mail: ykayashi@u-shizuoka-ken.ac.jp found that the survival of adult flies declined with the number of days after exposure to $\mathrm{H}_{2} \mathrm{O}_{2}$ (Fig. 1), suggesting that the oxidative stress response in adult Drosophila is impaired with age. This study demonstrated that the use of Drosophila enables the investigation of factors involved in aging-associated changes in response to oxidative stress.

Peroxiredoxins (Prxs) are a family of thiol peroxidases that decompose $\mathrm{H}_{2} \mathrm{O}_{2}$ and organic hydroperoxides (8). Human Prx3 (PRDX3) localizes to the mitochondrial matrix and displays strong reactivity to $\mathrm{H}_{2} \mathrm{O}_{2}$ (3). A mitochondrial dysfunction causes an abatement in ATP production, oxidative damage and the induction of apoptosis, all of which are involved in the pathogenesis of numerous disorders (11). Since all the members of PRDX family strongly scavenges $\mathrm{H}_{2} \mathrm{O}_{2}$ in a thioredoxin-dependent manner and PRDX3 is the only member of this family located in the mitochondria, PRDX3 is implicated in various age-related diseases that are considered to be caused either by accumulation of ROS or by insufficient ROS scavenging in mitochondria. However, actual involvement of PRDX3 in such agerelated diseases is poorly understood. A homologous gene of PRDX family was found in Drosophila, and Prx3, the Drosophila ortholog of PRDX3, was identified on the basis of a genome-wide search (6). 


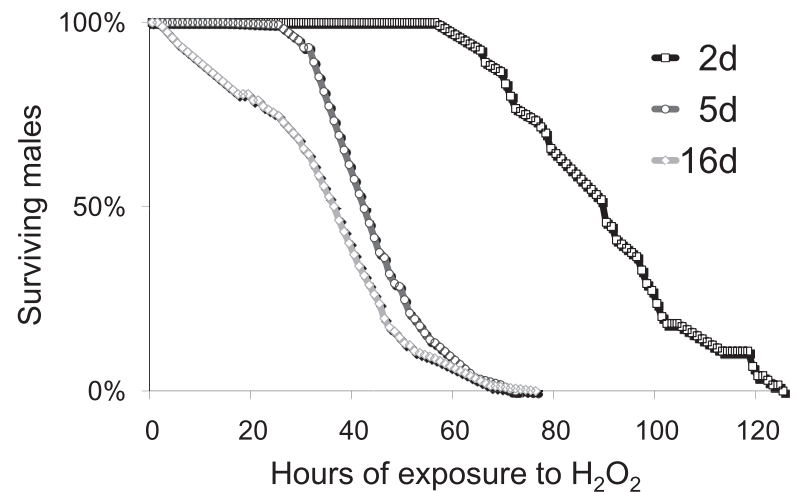

Fig. 1 Competence of the oxidative stress response depends on aging in adult Drosophila. Wild-type (Oregon-R) adult males (20 per vial) were directly exposed to $\mathrm{H}_{2} \mathrm{O}_{2}$ by adding $\mathrm{H}_{2} \mathrm{O}_{2}$ to the $1.3 \%$ sucrose medium. $2 \mathrm{~d}, 5 \mathrm{~d}$, and $16 \mathrm{~d}$ represent days after eclosion. Sample sizes (males): $2 \mathrm{~d}$, $\mathrm{n}=50 ; 5 \mathrm{~d}, \mathrm{n}=110 ; 16 \mathrm{~d}, \mathrm{n}=71$.

Although the role of Prx3 against stressors was evaluated in vitro using cultured cells (7) and its expression during phagocytosis of Candida albicans has been reported (13), its in vivo role in the fly is not clear.

To investigate the involvement of Prx 3 in the oxidative stress response in vivo, we used two transgenic fly lines (KD-1 and KD-2) in which the expression of the Prx3 was specifically and systemically reduced using the GAL4-UAS system and an RNAi approach. Both KD-1 and KD-2 transgenic fly lines contained an inverted repeat sequence of the Prx3 on chromosomes II and III, respectively. The RNAi effect became systemically active in all progeny following mating with the ubiquitous Gal4expressed line (Act5C). Quantitative RT-PCR revealed that the expression levels of the Prx3 in adult flies were significantly lower in the $\operatorname{Pr} x 3 \mathrm{KD}$ lines than in the control groups carrying Gal4 alone: the expression levels of the Prx 3 in the KD-1 and KD-2 mutants were $11 \%$ and $13 \%$ of those in the corresponding control lines, respectively (Fig. 2). We next investigated the resistance of the established $\operatorname{Pr} x 3 \mathrm{KD}$ lines against oxidative stress using 1-3-day-old male flies grown on agar plates containing $0.5 \% \mathrm{H}_{2} \mathrm{O}_{2}$. These $\operatorname{Pr} x 3 \mathrm{KD}$ lines were phenotypically similar to the wild-type lines, and the developmental rate, sex ratio, and lifespan were also comparable between the knockdown and wild-type lines (data not shown). Moreover, the ectopic expression of the Prx3 in vivo had no effect on lifespan (1). Under oxidative stress conditions, however, the median survival times of both KD lines was significantly lower than the control lines: the median

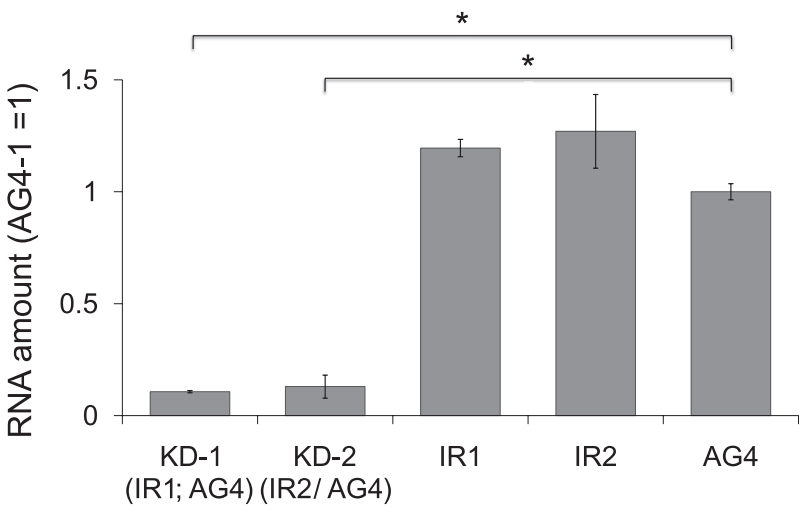

Fig. 2 Establishment of Prx3-specific knockdown lines. The fold RNA reductions of Act5C (ubiquitous promoter)-Gal4 > Prx3-anti1 (KD-1) and Act5C-Gal4 > Prx3-anti2 (KD-2) knockdown males are $11 \%$ and $13 \%$ compared to the control Act5C-Gal4/+ genotype (AG4), respectively. IR1 and IR2 are both transgenic fly lines that have the same Prx3targeted RNAi construct but integrated chromosome of its construct is different (IR1; 2nd chromosome, IR2; 3rd chromosome). Total RNA was isolated from 40 adults of each genotype and purified using RNAzol RT (Molecular Research Center, INC.). First-strand cDNA was synthesized using ReverTra Ace reverse transcriptase (Toyobo, Osaka, Japan) with $2 \mu \mathrm{g}$ of total RNA from each strain as a template and an oligo (dT) 20mer primer (Toyobo). Quantitative RT-PCR was performed on a ROTOR-GENE 6000 (Qiagen, Hilden, Germany) by following the manufacturer's instructions with SYBR green-based detection of PCR products. Data are expressed as mean \pm SE values. Significant differences are denoted as ${ }^{\star} P<0.005 . \mathrm{n}=5$.

survival times of the KD-1 and KD-2 lines were $84 \%$ and $79 \%$ of the corresponding control lines, respectively (Fig. 3). The survival rates under the oxidative stress conditions declined with age (the number of days after eclosion). The survival curves of the 1-3-day-old Prx3 KD lines were closer to those of the 10-day-old control lines than to those of the 1-3-day-old control lines.

These results suggest that the expression levels of the $\operatorname{Prx} 3$ play a role in the oxidative stress response. The Prx $3 \mathrm{KD}$ lines used in this study were more susceptible to oxidative stress, suggesting that Prx3 is involved in the oxidative stress response and the survival of Drosophila under drastic oxidative stress conditions. The control line Gal4/+ also showed decreased responsiveness to oxidative stress with age, similar to the results shown in Fig. 1. Our study also demonstrated that the responsiveness of the Prx3 KD lines to oxidative stress became more closer to that of the older control lines, suggesting the involvement of Prx3 in aging-associated changes in the responsiveness to oxidative stress. Quantitative RT-PCR was performed to investigate the changes 

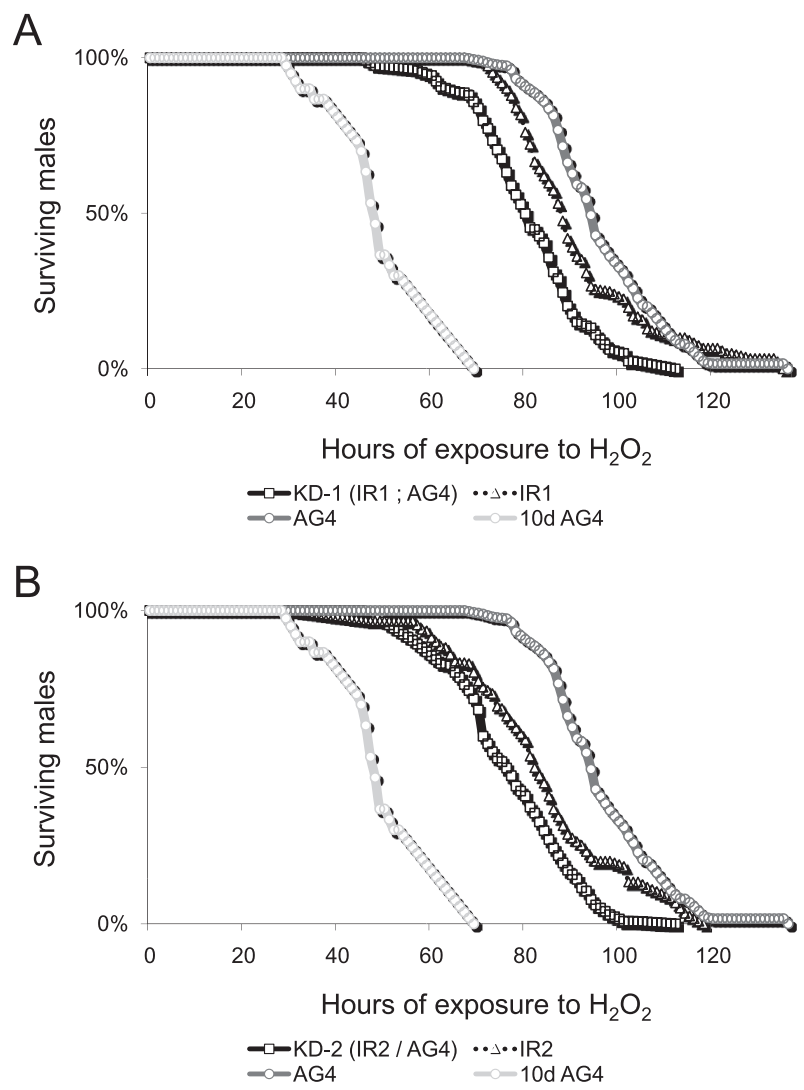

Fig. 3 RNAi-induced Prx3 knockdown mutants are sensitive to oxidative stress. A. Act5C (ubiquitous promoter)-Gal4 $>$ Prx3-anti1 (KD-1) flies had a 16\% shorter median lifespan than Act5C-Gal4/+ (AG4) controls $(P<0.05)$. The maximum survival time was reduced by $82 \%$. IR1; Prx3-anti1/+ control. Sample sizes (males): KD-1, $n=60$; IR1, $n=57$; AG4, $n=60 ; 10$ d AG4, $n=30$. B. Act5C-Gal4>Prx3-anti2 (KD-2) flies had a $21 \%$ shorter median lifespan than Act5CGal4/+ controls $(P<0.005)$. The maximum survival time was reduced by $82 \%$. IR2; Prx3-anti2/+ control. Sample sizes (males): KD-2, $\mathrm{n}=95 ; \mathrm{IR} 2, \mathrm{n}=60 ; \mathrm{AG} 4, \mathrm{n}=60 ; 10 \mathrm{~d}$ AG4, $n=30$.

in the expression levels of the $\operatorname{Prx} 3$ over time. It was revealed that its expression levels decreased in an age-dependent manner (Fig. 4). In particular, the expression of the Prx3 in 30-day-old males was significantly lower than that in 5-day-old males. This result indicates that the expression levels of the $\operatorname{Prx} 3$ may be crucial for the age-dependent competence of the oxidative stress response.

Human PRDX3 is a known target of the c-Myc oncogene (16). Although Prx3 is ubiquitously expressed in cells throughout development, enhancers that respond to the presence of drastic oxidative stress may exist in age-dependent manner. Identifying such regulators of Prx3 will clarify the detailed roles of Prx3 in aging-associated changes in the re-

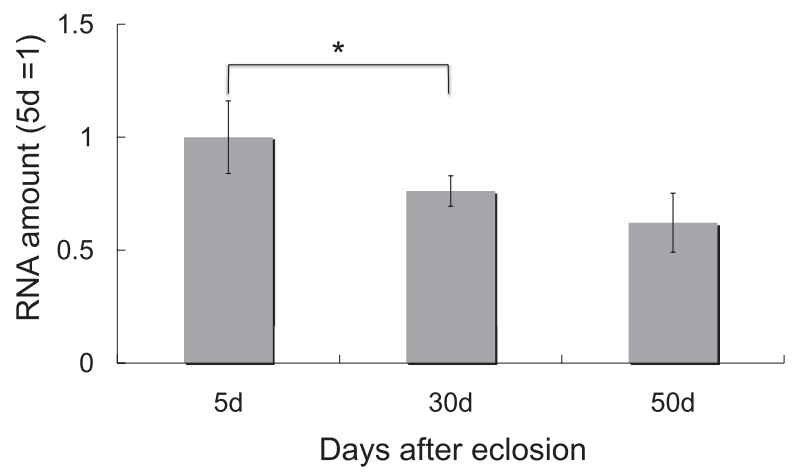

Fig. 4 The expression levels of Prx3 are deceased in an age-dependent manner. Data represent the fold increase in Prx3 mRNA levels, assessed using quantitative RT-PCR ( $5 \mathrm{~d}=1$, normoxia, standard food). The Prx3 mRNA level was $75 \%$ less on day 30 than on day 5 post eclosion $\left({ }^{*} P<\right.$ $0.05)$. RNA preparation and quantitative RT-PCR was performed as described in Fig. 2. Data are expressed as mean \pm SE values. $n=5$.

sponse to oxidative stress.

\section{Acknowledgements}

The following stocks were provided as generous gifts from S. Hirose and N. Fuse at the National Institute of Genetics, Japan: wild-type Oregon- $R, y^{l}$ $D f(1) w^{67 c 1}$; Actin5C-Ga14/TM6B (ubiquitous driver). The Prx3 RNAi lines $\left(w^{1118} ; 5826 R-1 / S M 1, w^{1118}\right.$; $5826 R-2)$ were obtained from the NIG-FLY stock center. This work was supported by Grant-in-aid for Young Scientist (B) (24700837) (to Y.K.) and Scientific Research from the Ministry of Education, Science, Sports, Culture, and Technology of Japan.

\section{REFERENCES}

1. Anderson PR, Kirby K, Orr WC, Hilliker AJ and Phillips JP (2008) Hydrogen peroxide scavenging rescues frataxin deficiency in a Drosophila model of Friedreich's ataxia. Proc Natl Acad Sci USA 105, 611-616.

2. Bulteau AL, Szweda LI and Friguet B (2006) Mitochondrial protein oxidation and degradation in response to oxidative stress and aging. Exp Gerontol 41, 653-657.

3. Cox AG, Peskin AV, Paton LN, Winterbourn CC and Hampton MB (2009) Redox potential and peroxide reactivity of human peroxiredoxin 3. Biochemistry 48, 6495-6501.

4. Fleming JE, Reveillaud I and Niedzwiecki A (1992) Role of oxidative stress in Drosophila aging. Mut Res 275, 267-279.

5. Miller RL, James-Kracke M, Sun GY and Sun AY (2009) Oxidative and inflammatory pathways in Parkinson's disease. Neurochem Res 34, 55-65.

6. Radyuk SN, Klichko VI, Spinola B, Sohal RS and Orr WC (2001) The peroxiredoxin gene family in Drosophila melanogaster. Free Radical Biol Med 31, 1090-1100.

7. Radyuk SN, Sohal RS and Orr WC (2003) Thioredoxin peroxidases can foster cytoprotection or cell death in response 
to different stressors: over- and under-expression of thioredoxin peroxidase in Drosophila cells. Biochem J 371, 743752.

8. Rhee SG, Chae HZ and Kim K (2005) Peroxiredoxins: a historical overview and speculative preview of novel mechanisms and emerging concepts in cell signaling. Free Radic Biol Med 38, 1543-1552.

9. Ristow M and Zarse K (2010) How increased oxidative stress promotes longevity and metabolic health: The concept of mitochondrial hormesis (mitohormesis). Exp Gerontol 45, 410-418.

10. Rossi P, Marzani B, Giardina S, Negro M and Marzatico F (2008) Human skeletal muscle aging and the oxidative system: cellular events. Curr Aging Sci 1, 182-191.

11. Sas K, Robotka H, Toldi J, and Vécsei L (2007) Mitochondria, metabolic disturbances, oxidative stress and the kynurenine system, with focus on neurodegenerative disorders. $J$
Neurol Sci 257, 221-239.

12. Stadtman ER (2006) Protein oxidation and aging. Free Radic Res 40, 1250-1258.

13. Stroschein-Stevenson SL, Foley E, O'Farrell PH and Johnson AD (2006) Identification of Drosophila gene products required for phagocytosis of Candida albicans. PLoS Biol 4, e4.

14. Versari D, Daghini E, Virdis A, Ghiadoni L and Taddei S (2009) The ageing endothelium, cardiovascular risk and disease in man. Exp Physiol 94, 317-321.

15. Wei YH, Lu CY, Lee HC, Pang CY and Ma YS (1998) Oxidative damage and mutation to mitochondrial DNA and agedependent decline of mitochondrial respiratory function. Ann NY Acad Sci 854, 155-170.

16. Wonsey DR, Zeller KI and Dang CV (2002) The c-Myc target gene PRDX3 is required for mitochondrial homeostasis and neoplastic transformation. Proc Natl Acad Sci USA 99, 6649-6654. 